\title{
Radiation protection design for the Super-FRS and SIS100 at the international FAIR facility
}

\author{
Ekaterina Kozlova ${ }^{1, *}$, Alexey Sokolov ${ }^{1}$, Torsten Radon $^{1}$, Rupert Lang $^{1}$, Inna Conrad ${ }^{1}$, Georg Fehrenbacher ${ }^{1}$, \\ Helmut Weick $^{1}$ and Martin Winkler ${ }^{1}$ \\ ${ }^{1}$ GSI Helmholtzzentrum für Schwerionenforschung GmbH, Planckstrasse 1, 64291 Darmstadt, Germany
}

\begin{abstract}
The new accelerator SIS100 and the Super-FRS will be built at the international Facility for Antiprotons and Ion Research FAIR. The synchrotron SIS100 is a core part of the FAIR facility which serves for acceleration of ions like Uranium up to $2.7 \mathrm{GeV} / \mathrm{u}$ with intensities of $3 \times 10^{11}$ particles per second or protons up to $30 \mathrm{GeV}$ with intensities of $5 \times 10^{12}$ particles per second. The Super-FRS is a superconducting fragment separator, it will be able to separate all kinds of nuclear projectile fragments of primary heavy ion beams including Uranium with energies up to $1.5 \mathrm{GeV} / \mathrm{u}$ and intensities up to $3 \times 10^{11}$ particles per second. During operation activation of several components, especially the production target and the beam catchers will take place. For handling of highly activated components it is foreseen to have a hot cell with connected storage place. All calculations for the optimisation of the shielding design of the SIS100, the Super-FRS and the hot cell were performed using the Monte Carlo code FLUKA, results are presented.
\end{abstract}

\section{Introduction}

FAIR [1, 2], Facility for Antiproton and Ion Research, will be a new international accelerator facility for the research with antiprotons and ions. It will be based upon an expansion of the GSI Helmholtz Centre for Heavy Ion Research which is situated in Darmstadt, Germany.

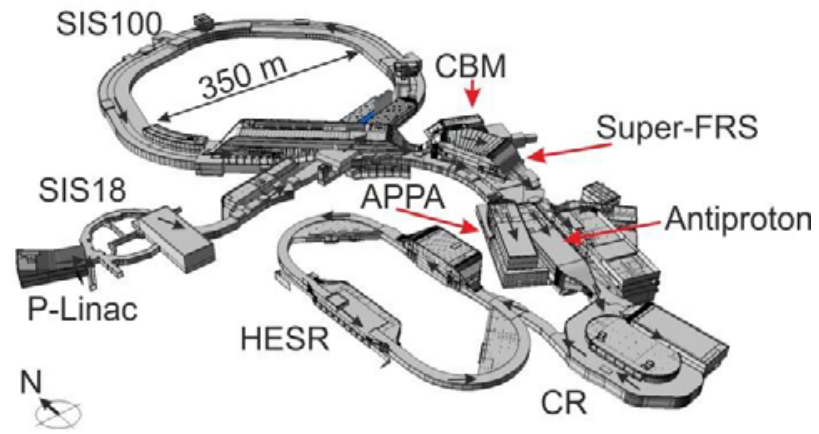

Fig. 1. The FAIR accelerator facility with the synchrotron SIS100, the experiments CBM, Super-FRS, APPA and PANDA, as well as the storage rings HESR and CR. The injected beam for FAIR comes from the GSI accelerator SIS18.

The existing accelerator facility at GSI after some appropriate modifications will be used as a preaccelerator for the FAIR facility. Extracted from the preaccelerator, high-intensity ion beams will be injected into the SIS100 and accelerated to the energy demanded by the experiments. Fig. 1 shows an overview of the FAIR facility with the SIS100 accelerator, the Super-FRS as well as the storage rings CR (Collector Ring), HESR (High Energy Storage Ring) and other experimental areas of different collaborations.

In this paper the radiation protection aspects of the SIS100 and the Super-FRS are discussed. They are the crucial parts of the facility in terms of radiation production.

\section{Prompt dose rate calculations}

The main role of radiation protection consists in avoiding unwanted exposure of man and environment to ionising radiation. The German radiation protection ordinance has defined dose limits which affect the design of the planned accelerator facilities. The effective dose rate must not exceed $1 \mathrm{mSv}$ per year for all areas and accelerator installations, which are within the premises and are freely accessible for workers and visitors. Since a working year is counted in this respect as 2000 working hours, the dose rate limit is $0.5 \mu \mathrm{Sv} / \mathrm{h}$. Thus for the shielding design the dose rate outside the shield must not be larger than this limit.

All calculations of the prompt dose rates for the SIS100 and Super-FRS were done with the Monte Carlo code FLUKA $[3,4]$. It is able to perform simulations of interactions with matter and propagations in matter for about 60 different particles. The transport of heavy ions was added in 2004 employing the RQMD and DPMJET models $[5,6]$. FLUKA has the capability of scoring fluences, energy depositions, activities, physical doses,

\footnotetext{
Corresponding author: e.kozlova@gsi.de
} 
etc. Dose rates were calculated with the help of fluenceto-dose conversion factors of [7, 8] linked to the code.

In the calculations the electro-magnetic cascade was switched off because photon and electron contributions to the integral dose value play a minor role in the concept of the shielding design here.

The use of biasing techniques was essential to obtain results with reasonable statistical significance. To apply Russian roulette and splitting at boundaries of certain volumes the shielding was divided into many layers of $50-\mathrm{cm}$ thickness in case of concrete.

\subsection{SIS100}

The SIS100 with circumference of $1083.60 \mathrm{~m}$ is five times longer than the injector SIS18 and can reach a magnetic rigidity of $\mathrm{B} \rho=100 \mathrm{Tm}$. It is located in the underground Tunnel 110 (shown on Fig.2). The SIS100 accelerates high energy and high intensity proton and ion beams by the synchrotron principle, in which charged particles are accelerated in high-frequency cavities and bend on a circular path with a constant radius by magnetic fields. The extracted primary particles from the SIS100 are protons and heavy ions up to uranium from which secondary particle beams of antiprotons and rare isotopes can be generated. These ions are applied for experiments in the fields of atomic, nuclear and plasma physics, and also for biophysics and materials research $[1,2]$.

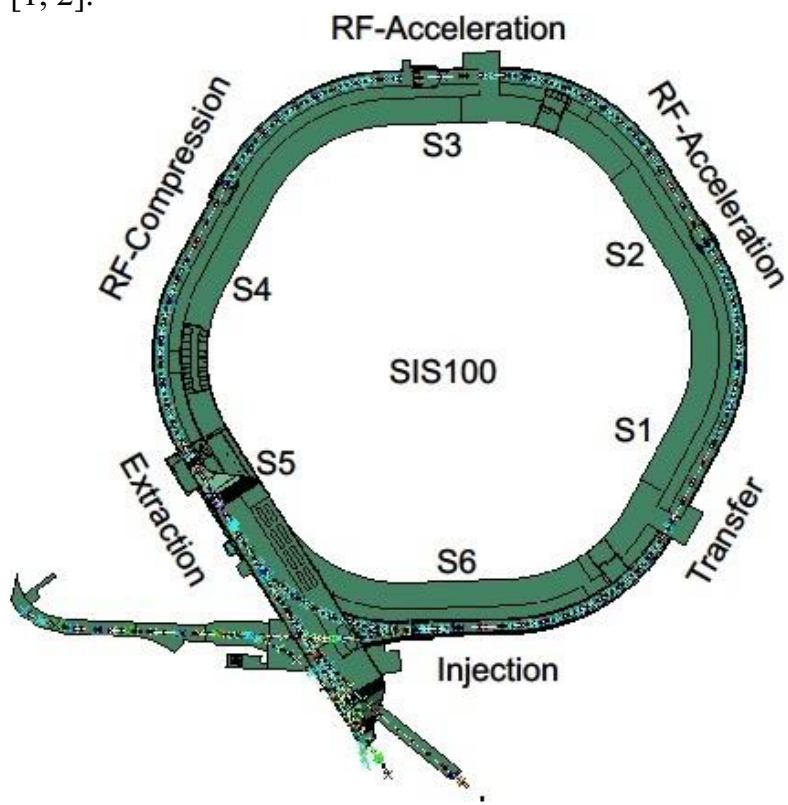

Fig. 2. Schematic layout of the SIS100 with injection, extraction, acceleration and beam compression on the straight sections of the sectors S1 - S6 along the circumference of $1083.60 \mathrm{~m}$.

The key parameters for the primary beams are summarised in Table 1:

Table 1. Maximum energies and intensities for accelerated particles in SIS100.

\begin{tabular}{|c|c|c|}
\hline Type of particle & Energy & Intensity $\left[\mathbf{s}^{-1}\right]$ \\
\hline Protons & $29.0 \mathrm{GeV}$ & $5 \times 10^{12}$ \\
\hline Uranium $^{28+}$ & $2.7 \mathrm{GeV} / \mathrm{u}$ & $3 \times 10^{11}$ \\
\hline
\end{tabular}

Such parameters can be achieved be applying new challenging features for the SIS100 synchrotron:

- operation at a very low base pressure of $10^{-12} \mathrm{mbar}$,

- careful control of beam losses,

- $\quad$ superconducting synchrotron magnet operation at a high ramp rate of $4 \mathrm{~T} / \mathrm{s}$,

- bunch compression of high intensity proton and uranium ion beams.

Tunnel T110, in which the SIS 100 is located, has many levels, three of them are underground (U10, U20 and U30), the other two (E10 and E20) are above. The level U30 basically consists of a ring-shaped beamline tunnel with the SIS 100 and a supply tunnel, separated by a total of $9.7 \mathrm{~m}$ wide shielding package as it is shown in Fig. 3 and Fig. 4. The space in the support tunnel is mostly designated for the electrical cabinets, which will provide the electrical support of the accelerator. The upper levels of the support tunnel house the extensive ventilation systems as well as supply rooms for cooling agents (water and liquid helium). Dedicated tracks connect the supply area with the accelerator. It is also planned to have three decay rooms in the support tunnel for the internal storage of not anymore needed or defective accelerator components. After sufficient decay time the activated components can be transported to the building for radioactive waste (Building 21). Building 21 is situated on the FAIR site and is used for the storage of the activated components and radioactive waste which will be produced during the facility operation.

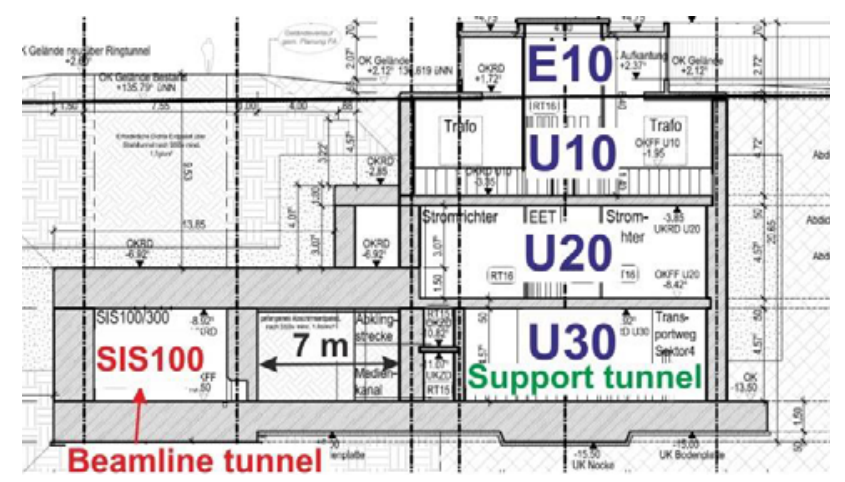

Fig. 3. Building plan for the SIS100 building showing cross section of the SIS100 beamline tunnel and the support tunnel. Both tunnels are divided by an earth layer.

For the calculation of the prompt dose rates the worst scenario with a $2.7 \mathrm{GeV} / \mathrm{u}$ uranium beam and local beam losses of $1 \times 10^{10}$ ions per second $(3 \%$ of the total beam intensity) are assumed. The result of the calculation is shown on Fig. 4. The dose rates above the soil layer on the public ground are below $50 \mathrm{nSv} / \mathrm{h}$. During synchrotron operation the dose rates in the supply tunnel and rooms above are less than $0.5 \mu \mathrm{Sv} / \mathrm{h}$. The access to the areas is allowed during beam operation. The calculations for $1 \times 10^{11}$ protons per second with the energy of $30 \mathrm{GeV}$ were also done. The resulting dose rates are close to the case of uranium losses. The assumed proton losses are approximately $2 \%$ of the total beam intensity, this should be very conservative for this part of 
the synchrotron ring as such losses are not expected according to beam loss estimations [9].

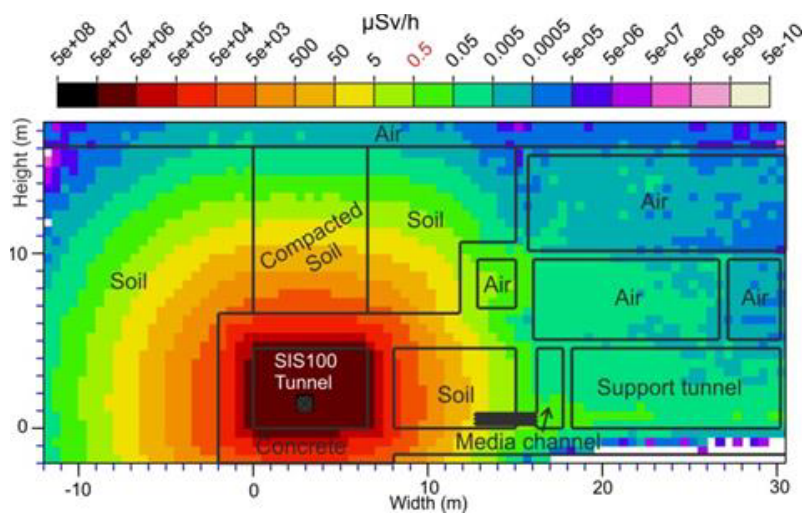

Fig. 4. Cross section of the SIS100 ring with calculated prompt dose rate in the SIS100 beam tunnel together with support tunnel, media channel and adjacent rooms.
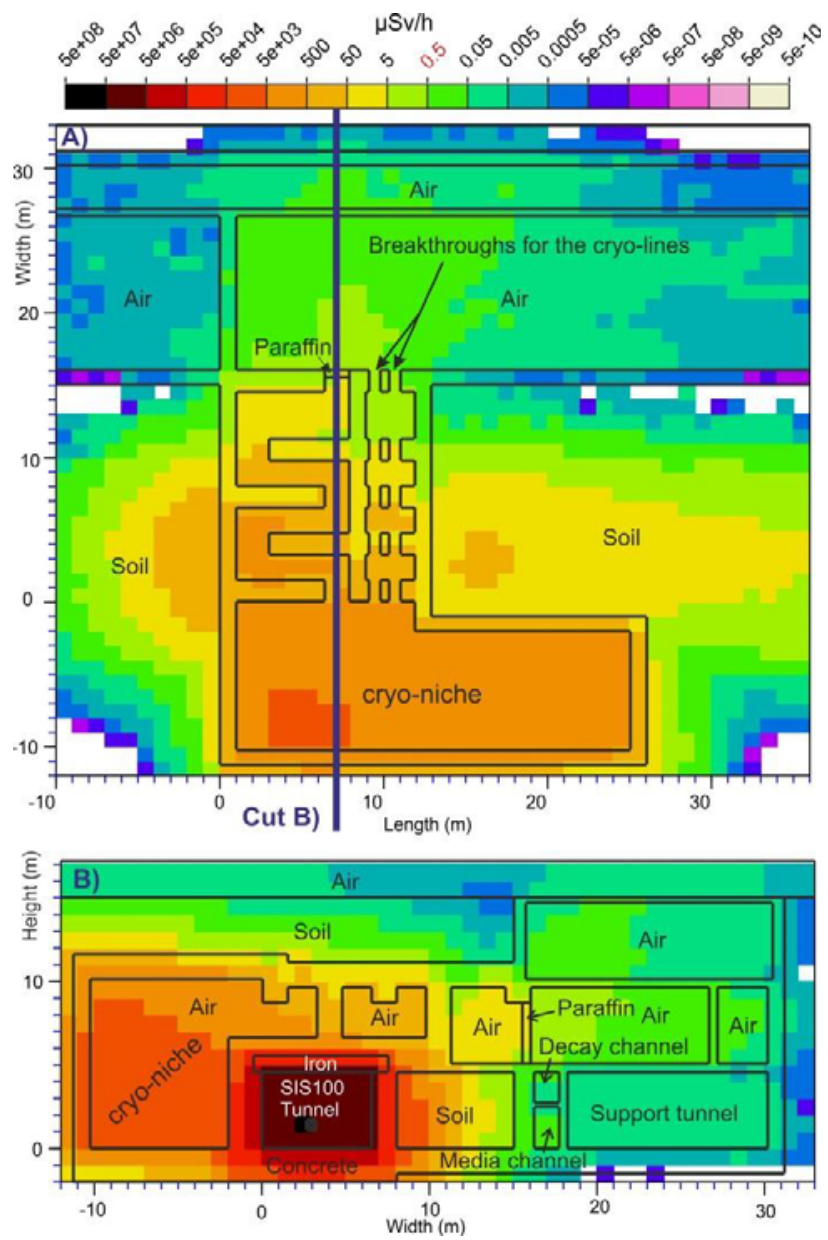

Fig. 5. Calculated prompt dose rate for the SIS100 ring with cryo-niche. A) Horizontal cross section of the labyrinth with breakthroughs for the cryo-lines. B) Cross section along the labyrinth, SIS100 beamline tunnel, support tunnel, cryo-niche and other rooms at different height levels.

On the outer side of the beamline tunnel three cryoniches are planned. The niches will provide the connection of the synchrotron via cryo-lines with the cryo-stations, which are situated on level U20. On Fig. 5 (A-top view) the cross section of the U20 level with the maze and additional access labyrinth for the cryo-lines are shown. Due to technical requirements of the cryosystem the cryo-lines should go straight to the cryoniches via breakthroughs in the additional access labyrinth. A location of 1 meter thick iron plate above the beamline tunnel and a 0.5 meter paraffin wall at the end of the labyrinth (as presented on Fig. 5 B) helps to reduce the dose rates above the building down to below $50 \mathrm{nSv} / \mathrm{h}$.

\subsection{Super-FRS}

The Superconducting magnetic FRagment Separator Super-FRS represents a major component of the planned FAIR [10]. The Super-FRS is an in-flight facility similar to the existing FRS at GSI [11] and serves for the production of rare isotopes at relativistic energies via fragmentation or fission in flight. The Super-FRS uses the $\mathrm{B} \rho-\Delta \mathrm{E}-\mathrm{B} \rho$ method, where a two-fold magnetic rigidity analysis is applied in front of and behind a shaped energy degrader. Compared to the existing separator the Super-FRS has more separation stages and twice larger magnet apertures made possible by use of superconducting coils.

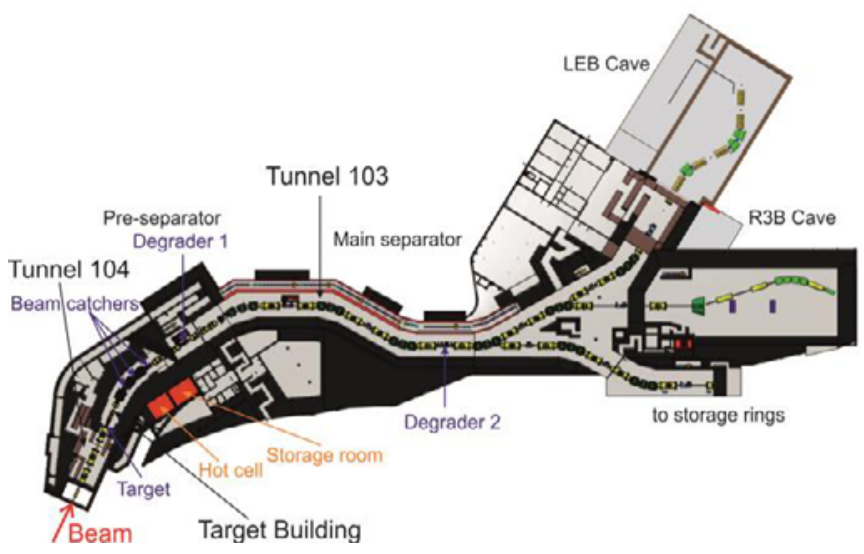

Fig. 6. Super-FRS layout, including the buildings and tunnels.

The SIS100 synchrotron will provide all kinds of heavy ion beams up to ${ }^{238} \mathrm{U}$ with maximum intensities of $3 \times 10^{11} / \mathrm{s}$ and beam energy up to $1.5 \mathrm{GeV} / \mathrm{u}$ for the SuperFRS. As it is shown in Fig. 6 the accelerated beam hits the production target after the focusing system. The created fragments leave the production target in forward direction, and then the isotope of interest can be selected depending on the setting of the separator.

For cleaner separation the Super-FRS applies the B $\rho$ $\Delta \mathrm{E}-\mathrm{B} \rho$ method twice in the pre- and in the mainseparator, both equipped with a degrader in the beam. After separation the rare-isotopes can be directed to one of the three experimental areas:

- The High-Energy Branch (HEB) where reactions of high-energy heavy nuclei will be investigated,

- The Low-Energy Branch (LEB) where properties of nuclei such as decay modes and energy levels can be explored using gamma and decay spectroscopy as well as trapped ions, 
- The Ring Branch (RB) where exotic nuclei are collected, cooled and stored in the Collector Ring CR. In the CR masses and life-times of unknown nuclei will be measured [2].

The production of rare isotopes in the target by fragmentation of heavy nuclei is accompanied by creation of neutrons of similar high velocities as that of projectile $[12,13]$. In Fig. 7 the pre-target focusing system of the ion-beam coming from the SIS 100 is shown, followed by the Super-FRS production target. The total beam losses can amount to $1 \times 10^{8}{ }^{238} \mathrm{U} / \mathrm{s}$ in the quadrupoles before the target in addition to the unavoidable beam losses in the target $\left(3 \times 10^{11238} \mathrm{U} / \mathrm{s}\right)$. The beam energy is $1.5 \mathrm{GeV} / \mathrm{u}$. The dose rates around the quadrupole triplet just in front of the production target are still high due to the strong neutron backscattering from the production target. For the access to the tunnel an entrance maze is foreseen. It is designed in such a way, that dose rates outside of the shielding and close to the maze in the stairwell, during Super-FRS operation are below $0.5 \mu \mathrm{Sv} / \mathrm{h}$.

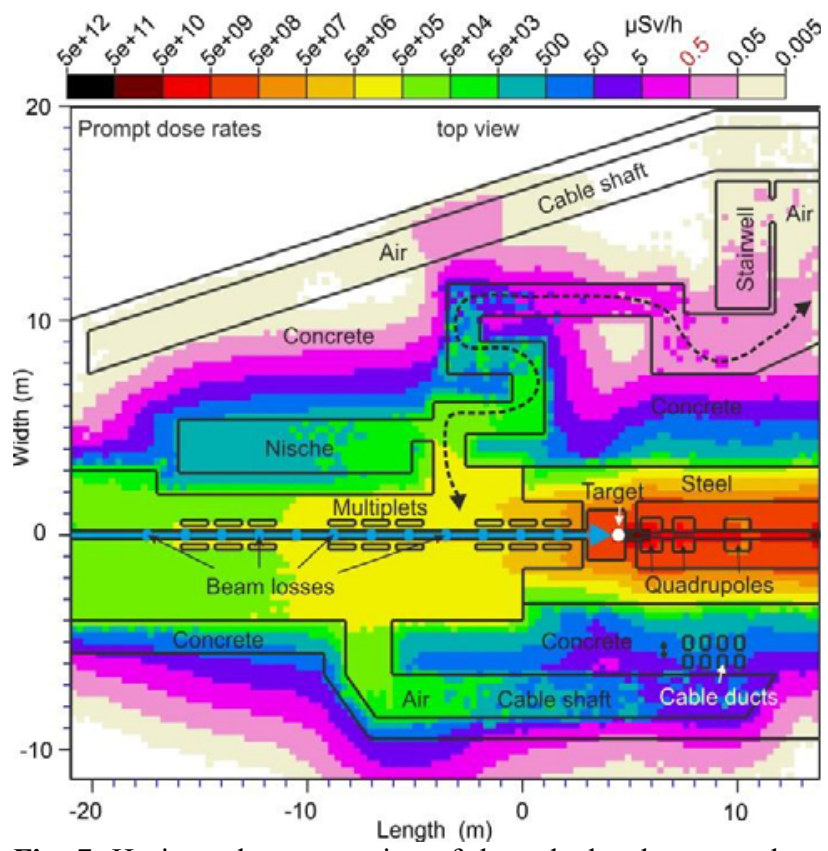

Fig. 7. Horizontal cross section of the calculated prompt dose rate distribution in the area upstream of the Super-FRS production target. The focusing system is situated in Tunnel 104, the production target is located inside Building 18 .

In the target and beam catchers area the shielding concept foresees a close compact shielding of the beamline to reduce the overall volume required due to the vicinity of other beamlines of the FAIR complex. To achieve a compact shielding, a multi-layer shield of steel followed by concrete is used. In the inner steel layer the high-energy neutrons are attenuated by elastic scattering and inelastic interaction (spallation reactions). In the outer regions of this steel layer the dose rate is dominated by contributions of low-energy neutrons and in the concrete region low-energy neutrons are absorbed due to the elastic scattering effect of hydrogen contained in the concrete. In Fig. 8 the distribution of dose rates towards the vertical direction in the plane intersecting the beam catchers is shown. The beam catchers are foreseen to absorb the primary beam at positions where its diameter is larger than at the target. In total six of these beam catchers are planned [12]. They are located in the first half of the Super-FRS pre-separator always following one of the $11^{\circ}$ dipole magnets as it is shown in Fig. 6 . Depending on the experiment each beam catcher can absorb up to $85 \%$ of the non-interacted primary beam. In Fig. 8 the beam line is surrounded by the steel core and followed by the concrete shielding. The dose rates outside the shielding are less than $0.5 \mu \mathrm{Sv} / \mathrm{h}$. The drives, connections and supplies for the target, beam catchers and many other components are done from above through the iron shielding. Therefore, on top of the iron shielding a service tunnel is provided for faster access. Otherwise the whole area is covered with a mobile ceiling consisting of 5 layers of concrete bars that can be taken off by the hall crane of the building.

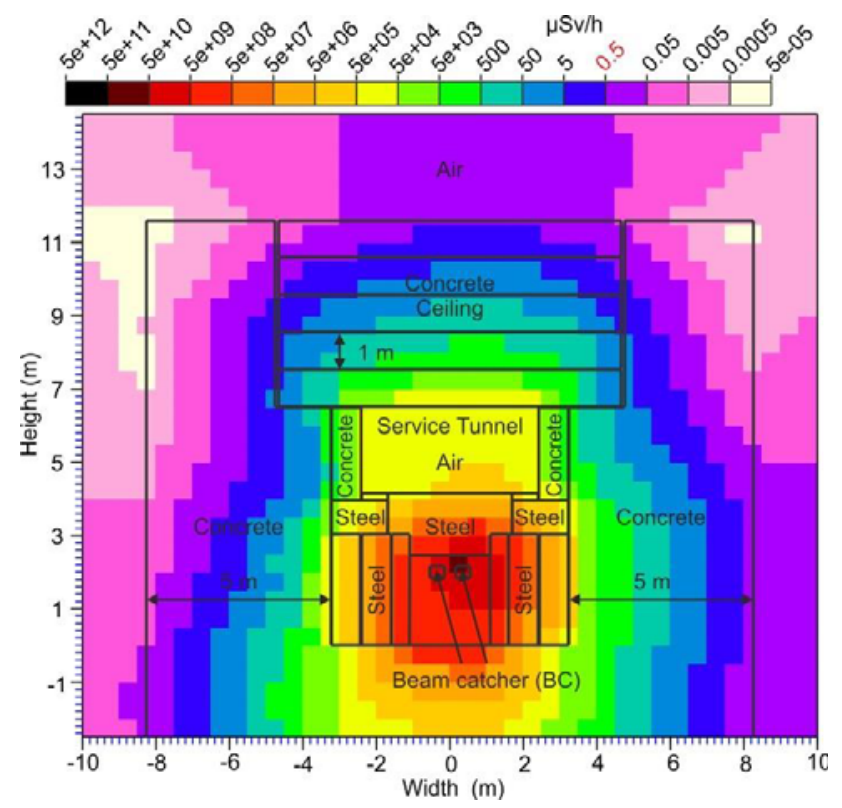

Fig. 8. Prompt dose rate calculations for $1.5 \mathrm{GeV} / \mathrm{u}$ Uranium beam of intensity of $3 \mathrm{E} 11 \mathrm{U} / \mathrm{s}$ in a cross section perpendicular to the beam at the beam catcher position.

As all primary beam and also the majority of the created fragments will be stopped in the target and beam catchers area the following part of the separator can be in an open tunnel with concrete shielding or a combination of concrete with soil as shown in Fig. 9 for the main separator. For the calculations of the dose rates in the main separator a ${ }^{114} \mathrm{Pd}$ beam as a typical fragment of the primary uranium beam was taken. ${ }^{114} \mathrm{Pd}$ will have energies up to $1.3 \mathrm{GeV} / \mathrm{u}$ and intensities up to $5.5 \times 10^{10}$ ions/s. The ions interact with two energy degraders and two slits. After a degrader ions have an energy lowered by a different amount depending on their atomic number and after the following separator stage the unwanted elements can be stopped in the thick tungsten slits. The second stage of the pre-separator and main separator is located in Tunnel 103.

In Fig. 9 all beam losses are marked. The number of ions hitting a degrader, due to their function, is higher 
than on the slits. If Degrader 1 is hit by $3 \times 10^{10} \mathrm{ions} / \mathrm{s}$ and Degrader 2 by $10^{10}$ ions $/ \mathrm{s}$, in the front slits $10^{10} \mathrm{ions} / \mathrm{s}$ and in the back slits $5 \times 10^{9}$ ions/s are deposited. As the ions are not lost in the aluminium degraders plates but only converted to some fraction or produce secondaries in the material in the FLUKA simulation they were modelled as plates of maximal useful thickness in a beam. The shielding in the Tunnel 103 is a composition of concrete walls with a thickness of $0.5 \mathrm{~m}$ and $2.5 \mathrm{~m}$ of compacted soil in between, followed by at least $7 \mathrm{~m}$ of soil outside the building. Along the main tunnel with the separator there is the supply tunnel with control racks for the cryoand vacuum support, data acquisition, quench detection and protection electronics, etc. The supply tunnel is subdivided from the main tunnel by a concrete wall with $0,5 \mathrm{~m}$ thickness.

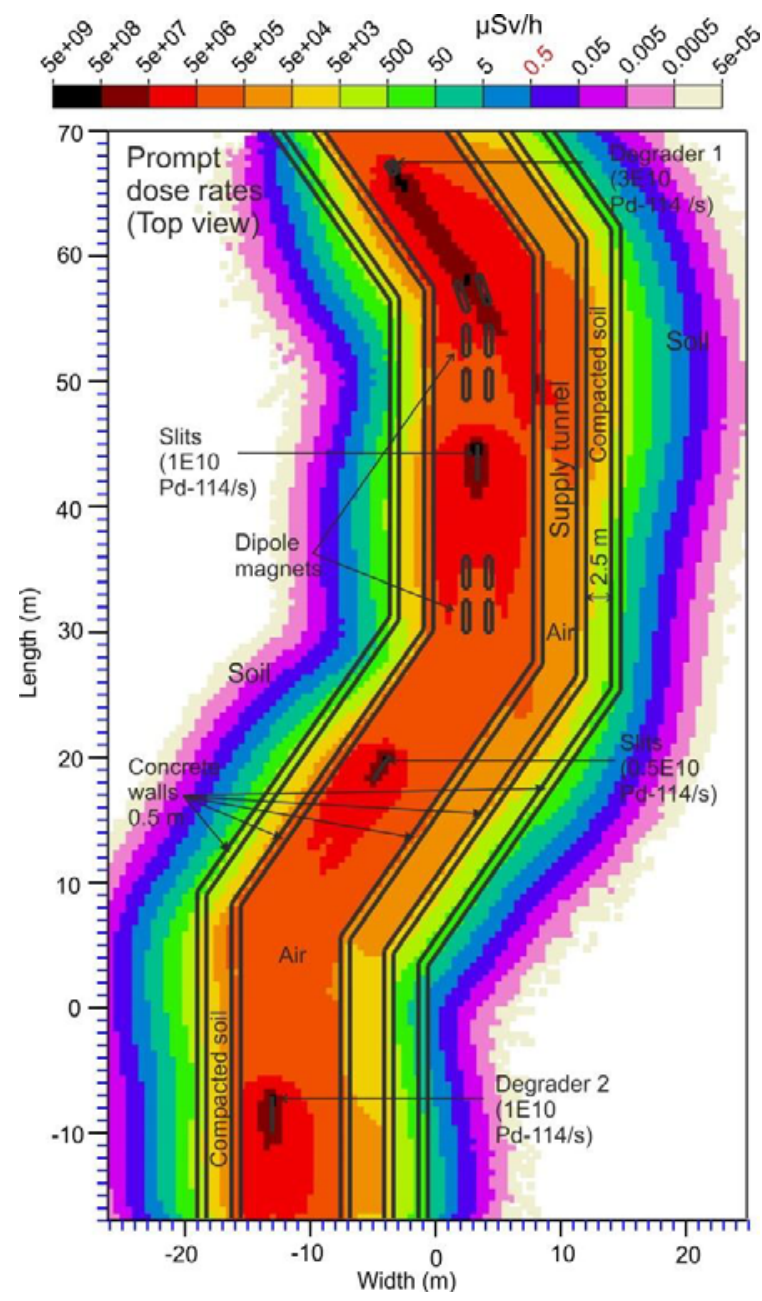

Fig. 9. Horizontal cut showing the prompt dose rate distributions in Tunnel 103 with four interaction points, two degraders and two slits.

\section{Hot cell}

The operation of the Super-FRS is inevitably associated with significant radioactive activation of components and targets where the primary beam interacts directly with materials. Activated components which have to be exchanged or maintained after an irradiation period cannot be handled by personnel directly. Therefore, it is obligatory to have a hot cell for handling these components using remote handling methods.

The use of the hot cell is planned as follows: The component (e.g. the production target) will be moved from the beam line into a shielding flask, which - on its part - will be moved to the roof position of the hot cell. The component within the flask will be lowered to the handling position in the hot cell, where it can be repaired or cut, separated and packed in barrels and moved over to a shielded storage position for radioactive decay (see Fig. 10). Maintenance work of the components will be carried out by means of remote manipulators. The hot cell with the storage place will be situated close to the Super-FRS target area as indicated in Fig. 6. The shielding layout of the hot cell was developed on the basis of activation calculations for components of the Super-FRS. The calculated radionuclide distributions within the components were used as radiation sources for estimating the shielding of the transport flask, the hot cell and the storage places in the neighbouring cell. The shielding design was done by using the FLUKA code in two steps. In the first step a simulation of production of the radioactive nuclides with build-up and decay for a certain irradiation pattern and different cooling times were performed. The simulations of the radioactive decay and the transport of the produced radiation were done in the second step with a new shielding geometry.

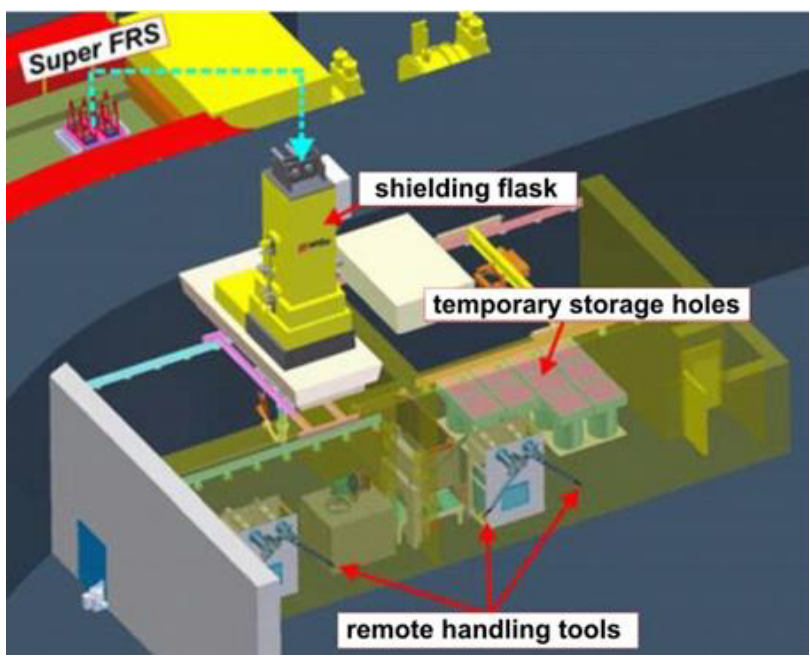

Fig. 10. Layout of the Super-FRS target and beam catchers area with the hot cell and the storage cell.

The largest and the most activated parts that have to be handled with the shielding flask are the beam catchers $[12,13]$. Fig.11 shows as an example of the plug with the beam catcher. Beryllium and graphite beam absorbers are used to stop the heavy-ion beam or to convert it to lighter fragments and to absorb most of the beam energy. The beryllium absorber is for the slow beam extraction and graphite absorber is for the fast beam extraction. The beam absorbers are coupled to a copper heat sink and mounted to an iron shielding plug with a size of $60 \mathrm{~cm} \mathrm{x}$ $80 \mathrm{~cm} \mathrm{x} 174 \mathrm{~cm}$ with drives, vacuum seals and feedthroughs on top. The total weight that has to be handled by the overhead crane is $7.5 \mathrm{t}$. 
After a long production period at maximum uranium beam intensity the activation reaches up to $3 \times 10^{13} \mathrm{~Bq}[12$, 13]. To obtain a realistic scenario for the estimation of the required wall thickness of the shielding flask, in a first step the beam catcher and plug were irradiated including the shielding environment of the Super-FRS. All information on the produced radionuclides is written to external files via a FLUKA user-routine (usrrnc.f). In the second step the beam catcher and plug are moved in the shielding flask and the information on radionuclides is read in from the file created in the first step via another user-routine (source.f). This method provides an accurate distribution of the nuclides in the device. The result is presented in Fig. 12. As realistic scenario, 3 days after four periods of 90 days of irradiation with an intensity of $3 \times 10^{11} / \mathrm{s}$ with a cooling time of 120 days between the blocks are assumed. In the example case only the graphite part was irradiated. The dose rates outside the shielding flask are below $10 \mu \mathrm{Sv} / \mathrm{h}$. During the transport of the activated components in the flask to the hot cell the target hall is specified as a controlled area.

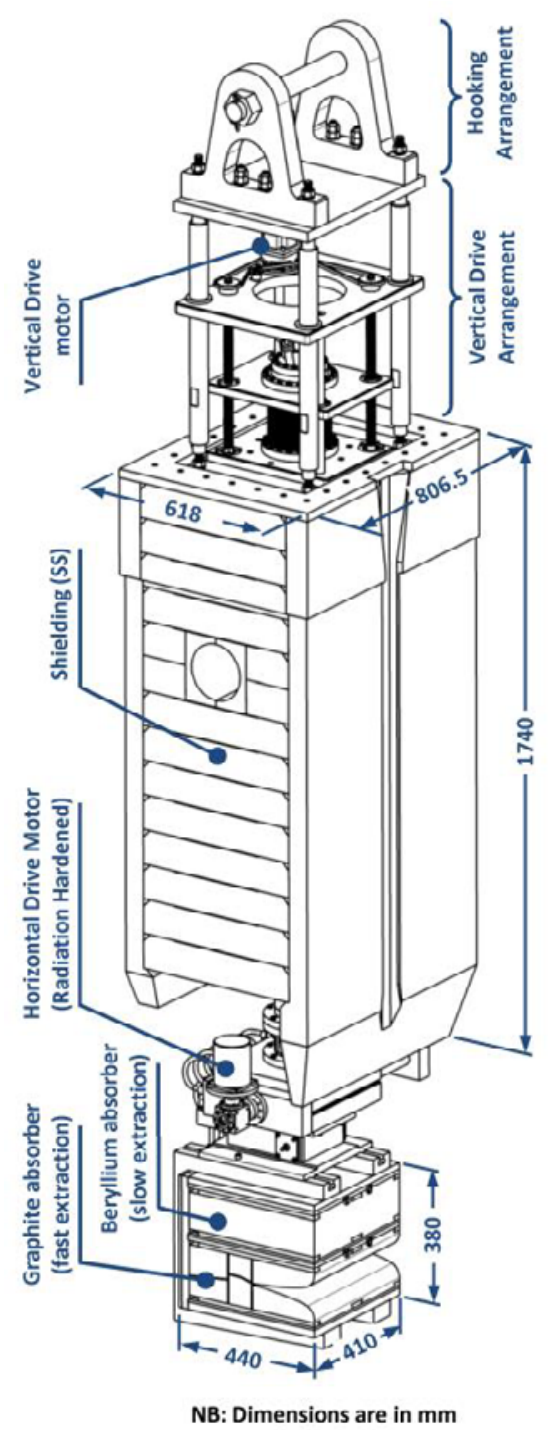

Fig. 11. Drawing of the beam catcher shielding plug. At the bottom the beam catcher is mounted, on top are the drives and a hook for removing. The catcher consists of the graphite and beryllium absorber.
The two step method was also applied for the optimization of the wall thickness in the hot cell. The activated beam catcher with the plug was situated in the center of the hot cell. In this case to achieve the dose rate outside the shielding below $0.5 \mu \mathrm{Sv} / \mathrm{h}$ at the concrete wall with a thickness of 1 meter is required.

For the design of the storage place also the activated beam catchers were taken in the calculations. It is foreseen to have 20 places for the 200-1 barrels with two levels (10 barrels on each level). In Fig. 13 four activated beam catchers after cooling times of 1 week, 1 month, 0.5 year and 1 year are situated in the storage holes with a concrete wall and iron cover. It is seen from Fig. 13 that the shielding wall thicknesses are sufficient to lower the dose rates outside the storage place to an appropriate level. The holes are situated in the storage room with the concrete wall thickness of $1 \mathrm{~m}$. Thus the dose rates outside the storage room will be below $0.5 \mu \mathrm{Sv} / \mathrm{h}$.

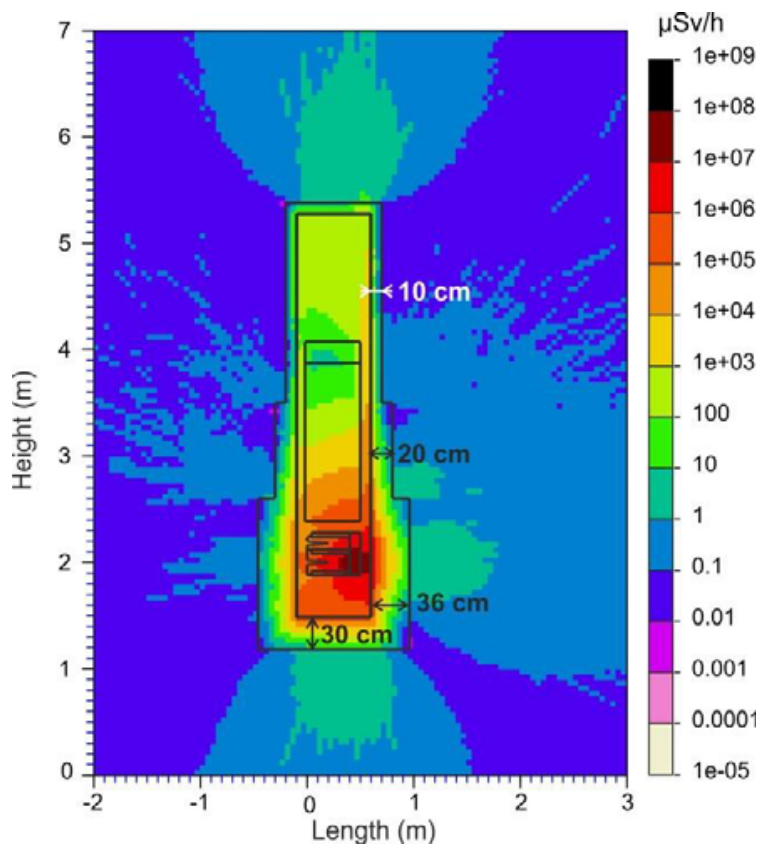

Fig. 12. Residual dose rates of the beam catcher und plug in the shielding flask.

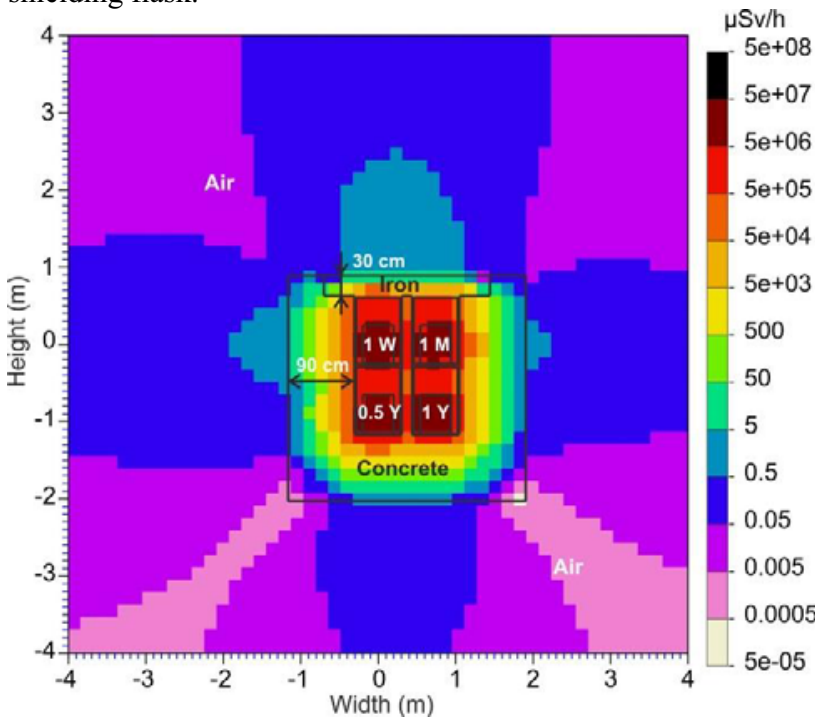

Fig. 13. Residual dose rates for the storage place. The four activated beam catchers are situated in the holes 


\section{Conclusions}

This paper presents the radiation protection studies for the SIS 100 and Super-FRS including the hot cell. Monte Carlo calculations were used for approving the shielding thickness and estimation of the propagation of neutrons through the mazes, shafts, cable ducts in the accessible areas. The calculations show that the shielding design for the SIS100 and Super-FRS is adequate from a radiation protection standpoint. The design value, to keep dose rates below $0.5 \mu \mathrm{Sv} / \mathrm{h}$ outside of the shielding and in all supervised areas nearby, is achieved.

A set of FLUKA calculations for the hot cell, the storage place and the flask was also performed to assess the residual dose rates inside and outside the shielding. In this case the calculations were done with a two steps method. In the first step the activation of some components and targets after several periods of SuperFRS operation and for different decay times was estimated. In the second step the residual dose rates for the hot cell, the storage place and the shielding flask from the most activated components, beam catchers, were calculated. As the result a suitable shielding design was developed on the basis of the FLUKA calculations.

The engineering design of Beam Stoppers for Super-FRS in FAIR project is currently ongoing at CSIR-CMERI, Durgapur, India under DST-DAE grant (GAP-098212) through BI-IFCC, Govt. of India. The authors acknowledge the contribution of Dr. Avik Chatterjee and Abhijit Mahapatra, Advance Design and Analysis Group, CSIR-CMERI for their contribution in engineering design.

\section{References}

1. An International Accelerator Facility for Beams of Ions and Antiprotons, Conceptual Design Report, GSI November 2001.

2. FAIR Baseline Technical Report, Darmstadt (2006). (https://www-alt.gsi.de/documents/DOC-2006-Jul40-1.pdf)

3. A. Fassò, A. Ferrari, J. Ranft, and P.R. Sala, FLUKA: a multi-particle transport code, CERN 2005-10 (2005), INFN/TC_05/11, SLAC-R-773

4. A. Fassò, A. Ferrari, S. Roesler, P.R. Sala, G. Battistoni, F. Cerutti, E. Gadioli, M.V. Garzelli, F. Ballarini, A. Ottolenghi, A. Empl and J. Ranft, The physics models of FLUKA: status and recent developments, Computing in High Energy and Nuclear Physics 2003 Conference (CHEP2003), La Jolla, CA, USA, March 24-28, 2003, (paper MOMT005), eConf C0303241 (2003), arXiv:hep$\mathrm{ph} / 0306267$

5. H. Sorge, Flavor production in $\mathrm{Pb}(160 \mathrm{~A} \mathrm{GeV})$ on $\mathrm{Pb}$ collisions: Effect of color ropes and hadronic rescattering, Physical Review C 52 (1995) 32913314.

6. J. Ranft, Phys. Rev. D51 (1995) p.64; New features in DPMJET version II.5, hep- ph/9911213 and DPMJET version II.5, code manual, hep$\mathrm{ph} / 9911232$.
7. International Commission on Radiological Protection, "Conversion Coefficients for use in Radiological Protection against External Radiation", ICRP Publication 74, Pergamon Press (1996).

8. A. Ferrari and M. Pelliccioni, Fluence to Dose Equivalent Conversion Data and Effective Quality Factors for High Energy Neutrons, Rad. Prot. Dosimetry 76 (1998) 215.

9. Beam Loss in SIS18/100/300/HEBT. Status 24.1.2009 - Version 7.0. FAIR Synchrotrons.

10. H. Geissel, M. Winkler, H. Weick, et al., Technical Design Report on the Super-FRS, 2008

11. H. Geissel, et al., The GSI projectile fragment separator (FRS): a versatile magnetic system for relativistic heavy ions, Nucl Instr. And Meth. in Phys. Res. B 70, 286-297 (1992)

12. E. Kozlova, H. Weick et al., Layout of the SuperFRS target hall, Nucl Instr. And Meth. in Phys. Res. B 266, 4275-4279 (2008)

13. T. Radon, E. Kozlova et al., Shielding of the target area of the fragment separator Super-FRS. Int. Conf. on Radiation Shielding (ICRS-11) 168, 492-496 (2009) 